\title{
Hepatic hydrothorax occurring rapidly after manual abdominal compression
}

\author{
Kazufumi Dohmen, Hirofumi Tanaka, Masatora Haruno, Yoshiyuki Niho
}

Kazufumi Dohmen, Hirofumi Tanaka, Masatora Haruno, Yoshiyuki Niho, Internal Medicine, Chihaya Hospital, Fukuoka 813-8501, Japan

Correspondence to: Dr. Kazufumi Dohmen, Internal Medicine, Chihaya Hospital, 2-30-1 Chihaya Higashi-ku, Fukuoka 813-8501, Japan.dohmen-kzfm@cool.odn.ne.jp

Telephone: +81-92-6612211 Fax: +81-92-6830411

Received: August 22, 2007 Revised: September 10, 2007

\begin{abstract}
Hepatic hydrothorax is a relatively infrequent but potentially serious complication of liver cirrhosis that often causes respiratory dysfunction. Several hypotheses for the development of hepatic hydrothorax have been suggested to explain a transdiaphragmatic shift of ascitic fluid through small defects between the peritoneal cavity and the pleural space. However, the rapid development of hydrothorax within several hours is seldom encountered. In addition, the causal factors for rapid passage of ascitic fluid into the pleural cavity are unknown. This report describes a patient with liver cirrhosis who suffered rapid development of a hydrothorax after manual compression of the abdomen.
\end{abstract}

(c) 2007 WJG. All rights reserved.

Key words: Hydrothorax; Liver cirrhosis; Abdominal compression

Dohmen K, Tanaka H, Haruno M, Niho Y. Hepatic hydrothorax occurring rapidly after manual abdominal compression. World J Gastroenterol 2007; 13(46): 6284-6285

http://www.wjgnet.com/1007-9327/13/6284.asp

\section{TO THE EDITOR}

A 63-year-old female with Child-Pugh grade C liver cirrhosis was admitted to Chihaya Hospital, Fukuoka, Japan because of ascitic fluid and hepatic encephalopathy. Physical examination revealed a markedly distended abdomen and peripheral edema. Her laboratory blood tests showed a white blood cell count of $9550 / \mathrm{L}$, hemoglobin of $7.6 \mathrm{mg} / \mathrm{dL}$, platelet count of $54000 / \mathrm{L}$, aspartate aminotransferase $39 \mathrm{IU} / \mathrm{L}$ (normal 7-38), alanine aminotransferase $16 \mathrm{IU} / \mathrm{L}$ (normal 4-43), gamma glutamyltransferase $45 \mathrm{IU} / \mathrm{L}$ (normal 16-73), alkaline

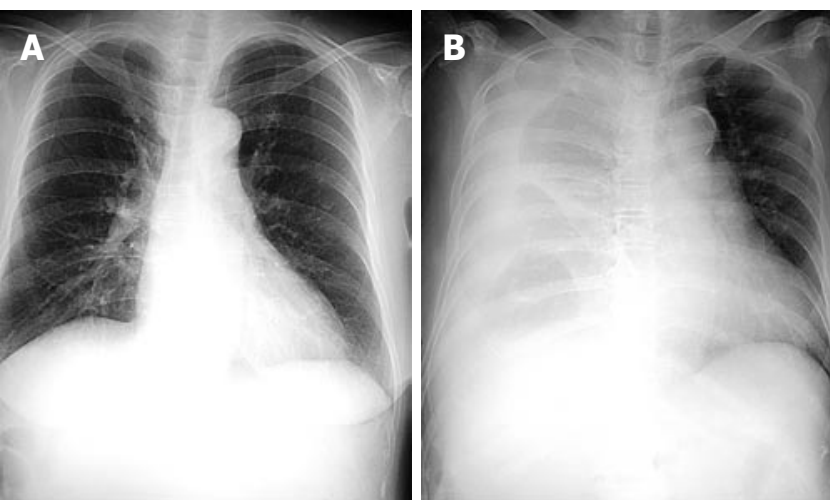

Figure 1 Chest X-ray. A: Before manual compression of the abdomen; $\mathbf{B}$ : on the next day, after manual compression of the abdomen.

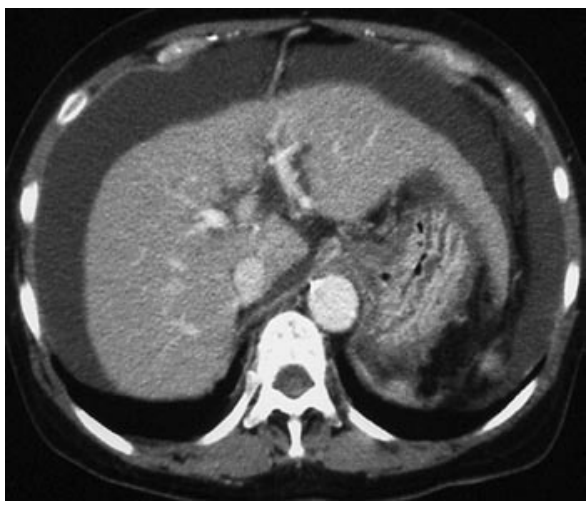

Figure 2 Abdominal computed tomography before manual compression of the abdomen shows liver cirrhosis with a massive volume of ascitic fluid.

phosphatase $600 \mathrm{IU} / \mathrm{L}$ (normal 104-338), serum albumin $2.0 \mathrm{mg} / \mathrm{dL}$ (normal 3.8-5.2), total bilirubin $1.5 \mathrm{mg} / \mathrm{dL}$ (normal 0.2-1.1), and serum ammonia $255 \mu \mathrm{g} / \mathrm{dL}$ (normal 30-86). Chest radiography showed no pleural effusion (Figure 1), while abdominal computed tomography imaging was characteristic of cirrhosis with a massive amount of ascitic fluid (Figure 2).

After admission, the patient was treated with branchedchain amino acid supplementation, diuretics and lactulose enema, in combination with antibiotics for ascites and hepatic encephalopathy. Hepatic encephalopathy had been well controlled; however, it manifested in association with constipation on fourteenth day after admission. Constipation was not relieved after the administration of laxative drugs and a lactulose enema. Therefore, 
manual abdominal compression was performed gently for approximately $1 \mathrm{~h}$ by a nurse to accelerate bowel movement. After that, a large amount of stool was evacuated and hepatic encephalopathy improved. However, during the following night, progressive dyspnea occurred and analysis of blood oxygen showed hypoxemia. Chest radiography on the next day revealed a massive right-sided pleural effusion (Figure 1), while ultrasonography showed that the ascitic fluid had disappeared. Pleural effusion obtained by thoracenteses showed a transudate, which indicated the rapid migration of ascitic fluid into the right hemithorax.

The transfer of large volumes of fluid from the abdomen to the pleural space through defects in the diaphragm can easily be understood. Once the diaphragmatic communications begin to leak fluid in response to abdominal pressure such as a cough or muscle strain, the combination of a hydrostatic gradient of negative intrathoracic pressure and positive intraabdominal pressure passively produces a unidirectional flow of ascitic fluid into the pleural space ${ }^{[1,2]}$. The occurrence of hydrothorax induced by tense and pro- longed abdominal compression has not previously been reported, although there have been a few reports demonstrating the actual condition of the rapid occurrence of hepatic hydrothorax ${ }^{[3]}$.

Interestingly, in this case with cirrhosis and a massive volume of ascitic fluid, the imaging data and clinical course strongly suggested that the rapid migration of ascitic fluid into the pleural space occurred due to the manual abdominal compression. Therefore, clinicians need to be aware of the possibility that massaging the abdomen can cause a hepatic hydrothorax in patients with a massive volume of ascitic fluid.

\section{REFERENCES}

1 Borchardt J, Smirnov A, Metchnik L, Malnick S. Treating hepatic hydrothorax. BMJ 2003; 326: 751-752

2 Al-sharif $\mathbf{H}$, Sharma S. Hepatic hydrothorax -- how would you manage it? Can Respir J 2005; 12: 440-442

3 Tsubouchi N, Hasuike S, Uto H, Kato J, Ido A, Hayashi $\mathrm{K}$, Tsubouchi H, Motoda M. Rapidly increasing hepatic hydrothorax induced dyspnea in two cases with liver cirrhosis. Acta Hepatol Jpn 2004; 45: 202-205

S- Editor Liu Y L- Editor Kerr C E- Editor Ma WH 